\title{
Investigation of Working Bodies of the Device for Separation of Fibers Suitable for Spinning from Cotton Waste
}

\author{
Avazbek Obidov, Mirzaolim Sultonov \\ Namangan Institute of Engineering and Technology, Namangan, Republic of Uzbekistan \\ Email: aobidov@list.ru
}

How to cite this paper: Obidov, A. and Sultonov, M. (2020) Investigation of Working Bodies of the Device for Separation of Fibers Suitable for Spinning from Cotton Waste. Engineering, 12, 893-905. https://doi.org/10.4236/eng.2020.1212063

Received: November 24, 2020

Accepted: December 27, 2020

Published: December 30, 2020

Copyright $\odot 2020$ by author(s) and Scientific Research Publishing Inc. This work is licensed under the Creative Commons Attribution International License (CC BY 4.0).

http://creativecommons.org/licenses/by/4.0/ (c) (i) Open Access

\begin{abstract}
It is known that fiber wastes (lint, down and seeds) produced at ginneries contain fibers that are suitable for spinning and can be used in industry, and their separation significantly increases the level of fiber production $(1.9 \%$ $2.5 \%)$. Based on these analyzes, the study aimed to create a new device that separates long fibers from lint and down. As a result, the amount of fiber output in the enterprise will increase and the enterprise will have significant economic benefits. In addition, the introduction of the device will prevent the addition of long fibers (longer than $16 \mathrm{~mm}$ ) that can be used in the textile industry to the waste. This article focuses on the creation of a fiber separation device suitable for the treatment and spinning of fibrous waste produced in ginneries. The study theoretically examined the main working bodies of the fiber separation device from waste. Theoretical research is devoted to the study of the strength of the main working body of the fiber separation device-the separating saw drum and its shaft. In the study, the sawdust drum is a more stressed steel coating, and it was found that the strength reserve of this drum is $\left[\delta_{\mathrm{T}}\right]=2.03$ (where $\delta_{\mathrm{T}}=0.8-2.5$ ) was found to be. As a result of calculating the resistance of the saw drum shaft to stiffness and vibration, it was determined that the shafts are resistant to vibration under periodic loading and that the oscillation frequency along its axis through the critical rotation frequency is $v_{c r}=10.3 \mathrm{Gts}$.
\end{abstract}

\section{Keywords}

Saw Drum, Device, Cotton Fiber, Fiber Waste, Down, Lint, Short Fiber, Guide, Diameter, Steel Coating, Strength, Strength Reserve, Shaft, Centrifugal Force, Cotton, Technology 


\section{Introduction}

The working parts of the device serve to grind, clean and separate industrially suitable, relatively long fibers from waste and short fibers from technological processes. These working parts must meet certain technical requirements of the technological process in which they are performed.

The following is a list of these technical requirements [1] [2]:

Working bodies should not damage fibrous materials as a result of dynamic shocks to the fibers during movement.

In the process of designing working bodies, when fastening saws, saw drums and guides, care must be taken to ensure that the air flow resulting from their rotational movement is evenly distributed over the width of the device.

Working bodies in direct contact with fibrous materials are subject to abrasion due to regular friction. Therefore, during the design process, special measures against abrasion of the working bodies by friction should not be neglected.

All working bodies projected on the device must be balanced in order to reduce the various voltages applied to the supports and to prevent vibration. The effect of working bodies on fibrous materials can be conditionally divided into three: mechanical, hydraulic and pneumatic effects. Among them, mechanical impact is common, and all working organs in the device are under this influence.

\section{Method}

\subsection{Investigating the Separation Drum Design and Its Robustness}

The diameter of the saw drum is also a key parameter, in the working bodies of fibrous waste processing equipment mainly 6 types of diameters from 200 to 610 $\mathrm{mm}$ are used.

At present, the optimal diameters of drums $(250,400,630 \mathrm{~mm})$ are used as a basis for the use of new devices in design constructions [3] [4] [5]. It is not recommended to use working bodies with a diameter of less than $250 \mathrm{~mm}$ for processing fibrous materials with a length of $20 \mathrm{~mm}$ and more. Because they are more likely to be wrapped in fibrous materials.

The working bodies produced in machine-building enterprises must be tested by a stand, in which the angular velocity of the test stand is $20 \%$ higher than the maximum speed of the device. In calculating the strength of the working bodies of the devices, taking into account the centrifugal force acting on them, the speed is calculated by setting the speed in the stand test in the enterprise [6] [7].

The saw drum (Figure 1) has a gauge diameter of $560 \mathrm{~mm}$, the saw blade is taped to the 4 steel cladding, the cladding is fastened to the 3,7 crossbars, the crossbars 7 are fastened to the shafts by means of a conical bushing 1, 6 through a 1-nut. Both sides of the drum are covered with shit $2,5 \mathrm{~s}$. The drum rotates on spherical roller bearings 12 and they are attached to the housing 11 . It is moved by means of pulley 8 , the shields are fastened with 10 bolts.

The crossbar holes are covered with a steel cladding wrapped with saw metal 
tape using bolts. The holes are drilled and made relatively light so that the steel cladding does not weigh down the device. In addition, the steel coating makes the outer surface of the drum look cylindrical. Steel plates are fastened to the sides of the cross. The load is fixed to the plate to ensure the dynamic balance of the drum between the discs.

The plates are fastened with angles-2. When the drum rotates, these angles create an air flow from the drum axis in the direction of its outer diameter, while at the same time preventing fibrous materials from entering the shafts.

The most stressful part in saw drums is the steel cladding. Steel cladding can be considered as multi-beam beams (Figure 2).

An example of this is the 1-1 cross-section, in which the bending moment is equal to:

$$
M_{\max }=0.107 q a^{2}+0.54 P a,
$$

where: $R$ is the centrifugal force acting on the saw;

$q$ is the intensity of the stress on the steel cladding;

$a$ is the distance between the bases.
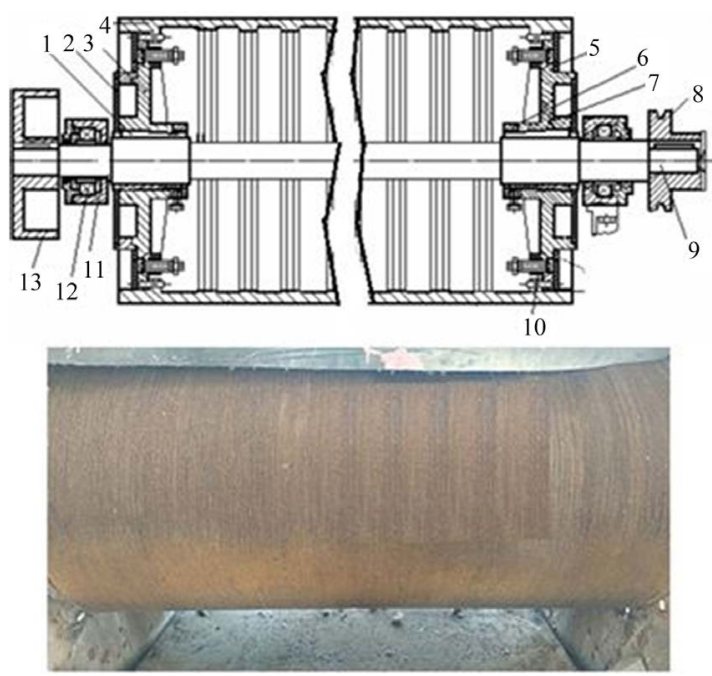

Figure 1. Saw drum. 1, 6-conical bushings, 2, 5-side steel cladding, 3, 7crosses, 4-steel cladding, 8-pulley, 9-shaft, 10-bolt, 11-bearing housing, 12-bearing.

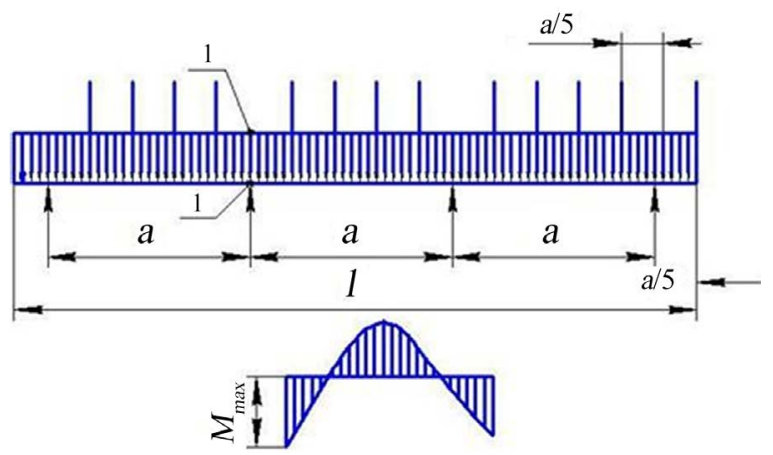

Figure 2. Schematic of the saw drum. 


$$
P=m \omega^{2} r, H
$$

where: $m$ is the surface mass of steel coatings and saw sets of $10 \mathrm{~cm}^{2}, \mathrm{~kg}$;

$\omega$ is the rated rotation speed of the saw drum (20\% higher than the working speed, $1 / \mathrm{s}$ );

$r$ is the distance from the center of gravity to the axis of the saw drum, $m$.

$$
q=\frac{P}{\ell} \mathrm{N} / \mathrm{m}
$$

where: $R$ is the centrifugal force, $\mathrm{N}$;

$l$-radius of the serrated drum, $\mathrm{m}$.

In that case

$$
\begin{gathered}
M_{\text {max }}=0.107 q a^{2}, \mathrm{~N} / \mathrm{m} \\
P=m \cdot \omega^{2} \cdot r_{k} ; q=R / L ; R=m \cdot \omega^{2} \cdot r_{P L} ; L=3 a+2 a / 5=3.4 \Rightarrow a=L / 3.4
\end{gathered}
$$

Maximum stress in steel cladding:

$$
\sigma_{\max }=\frac{M_{\max }}{W},
$$

where: $W$ is the moment of resistance in the dangerous section of the steel cladding.

A schematic of the hazardous cutting profile of the steel cladding is shown in Figure 3.

$$
W_{X}=\frac{d^{2}(c-b)}{6}=-H
$$

then: $H=0.92 \mathrm{~m}$.

$$
\delta_{\max } \leq[\delta] ;[\delta]=\delta_{T} /[n]
$$

Consistency reserve $[n]=1.3-1.5 ; \delta_{T}$-the strength limit is taken from the reference for the selected material [8] [9].

Taking into account the initially given sizes, we calculate the strength of the saw set drum:

$$
\begin{gathered}
m=3.0 \mathrm{~N} ; \omega=4801 / \mathrm{s} ; \quad r=0.05 \mathrm{~m} ; l=0.025 \mathrm{~m} ; \\
P=3 \times 400 \times 0.05=60 \mathrm{~N} / \mathrm{m}^{2} ; q=0.250 \mathrm{~m} ; a=0.870 \mathrm{~m} ; \\
L=3 \times 0.870+2 \times 0.870 / 5=2.61+0.348=2.95 ; R=30 \mathrm{~N} ; H=0.9 \mathrm{~m} ;
\end{gathered}
$$

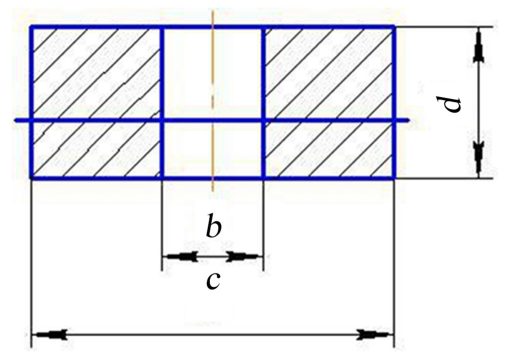

Figure 3. Hazardous cutting profile of steel cladding. 


$$
\begin{gathered}
M_{\text {max }}=0.107 \times 0.25 \times 0.87^{2}+0.54 \times 4 \times 0.87=0.02024+1.88=0.19 \mathrm{~N} / \mathrm{m} \\
q=\frac{60}{0.25}=240 \mathrm{~N} / \mathrm{m} \\
W_{X}=\frac{0.002^{2}(0.3-0.03)}{6}=0.15 \mathrm{~N} \\
\sigma_{\text {max }}=\frac{M_{\text {max }}}{W}=\frac{0.19}{0.15}=1.2
\end{gathered}
$$

$\left[\delta_{T}\right]=2.03$, in this $\left(\delta_{T}=0.8-2.5[10]\right)$.

As a result of calculating the strength of the saw drum, it is a steel coating that is under more stress, and it was found that the strength reserve of the drum is $\left[\delta_{T}\right]=2.03$ ( where $\left.\delta_{T}=0.8-2.5\right)$.

This means that the condition is met and the strength of the saw drum is ensured.

Figure 4 shows a graph of the relationship between the centrifugal force acting on the saw drum and the strength of the drum in the hazardous zone.

As a result of the study, a graph of the connection of the centrifugal force in the saw drum to the strength reserve in the drum hazardous zone was obtained.

\subsection{Investigation of the Strength of the Separating Drum Shaft of the Fiber Separation Device}

This method of calculating the saw drum shaft of a new device is based on the laws of material resistance, the diameter of the hazardous section of the shaft is determined on the basis of the given torque, and the given torque is determined on the basis of bending and twisting torques.

When selecting the initial calculation scheme of the shafts, the shaft supports can be of three different types: stationary hinged, movable hinged and clamped. In this case, it is recommended to take a fixed hinged support on the bearing under the influence of radial and longitudinal forces, and only under the influence of radial forces on a movable hinged support.

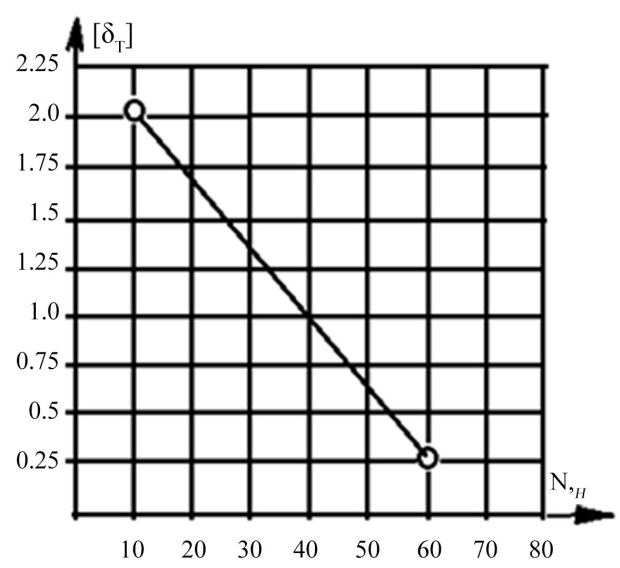

Figure 4. Graph of the connection of the centrifugal force in the saw drum to the stability reserve in the hazardous zone of the drum. 
In the defined method of calculating the strength of the shaft, in addition to the moments of influence, the safety factor for the hazardous section is determined by taking into account the accumulation of stresses in hazardous sections, the geometric dimensions of the shaft and the effect of surface cleanliness on stress values.

The shafts are rated for strength, durability and vibration resistance. The calculation of the strength of the shafts is done to prevent the formation of plastic deformation and premature breakage. It is known that such cases occur when the shafts are moving with a large load. The main reason for this is random factors and the start-up period. The purpose of the calculation of virginity is to determine the elastic deformation under the influence of load and compare it with the allowable value.

The purpose of calculating vibration resistance is to determine the vibration resistance of the shafts as a result of periodic loading. The calculation of the stiffness and vibration of the shafts is not shown here, as they fall into special cases (spindles of metalworking machines, long transmission shafts, etc.).

The strength of the shafts is one of the key factors that ensure the smooth operation of the fiber separation machine. The following steps are taken into account when calculating the strength of the shafts:

- determine the approximate diameter of the shaft on the basis of design calculations before creating the structure;

- development of shaft structure;

- determination of the voltage in the dangerous section of the shaft;

- to clarify the structure of the shaft, if necessary.

In the study, the shafts of saw drums are resistant to bending moment and torque, stiffness and vibration resistance.

A certain methodology was used to perform the calculations [10] [11] and a shaft model was created for small experiments.

The calculation of the strength of the shafts was performed in the following order:

By drawing a sketch of the shaft, an approximate calculation can be made using an empirical formula or by taking into account the torque effect by the following equation [12]:

$$
P_{u \kappa}=\frac{M_{u}^{2}}{\left[\frac{\pi d l^{2}}{12}+\mu_{1} d l\left(d+\frac{1}{2}\right)\right]^{2}}+\frac{M_{K \max }^{2}}{\left\{\frac{\pi d^{2} l \mu^{2}}{2}\right\}}
$$

In this case: $M_{u}$-the bending moment of the shaft;

$M_{K \max }=2 M_{K n n o}$-the moment of maximum and short-term impact;

$d$-the diameter of the hollow part of the shaft;

$\mu_{1}, \mu_{2}$-axial and rotational friction coefficients;

l-the length of the bricma-forming transition of the detail.

The contact pressure acting on the surfaces of the attached part in a tight fit is determined from the following equation: 


$$
P_{n}=\frac{\frac{\Delta}{d}}{X \frac{1}{E_{1}}\left(1-\mu_{1}\right)+\frac{1}{E_{2}}\left(\frac{1+K_{2}^{2}}{1-K_{2}^{2}}+\mu_{2}\right)}
$$

In this case: $K_{2}=\frac{r_{1}}{r_{2}}$-the inner and outer radii of the shaft cavity;

1) A variable section of the shaft on two supports on which the details are fastened,

2) Is a constant section of the equivalent shaft.

$X$-coefficient, $X=1$ can be seen as, because, $\frac{1}{d}>1$

$E_{1}, E_{2}$-modulus of viscosity of tsapfa and shaft material;

$\Delta$-tight transfer between details.

Based on the strength condition of the transitions between details:

$$
P_{n}>P_{u \kappa}
$$

Assuming that the elastic line of the shaft is sinusoidal, we write the following:

$$
y=f \sin \frac{\pi X}{l},
$$

in this: $y$-bending of the shaft in the $\mathrm{x}$ shear;

In Figure 5(a), we replace the variable cut beam $(b)$ with a permanent cut beam, resulting in:

(a)
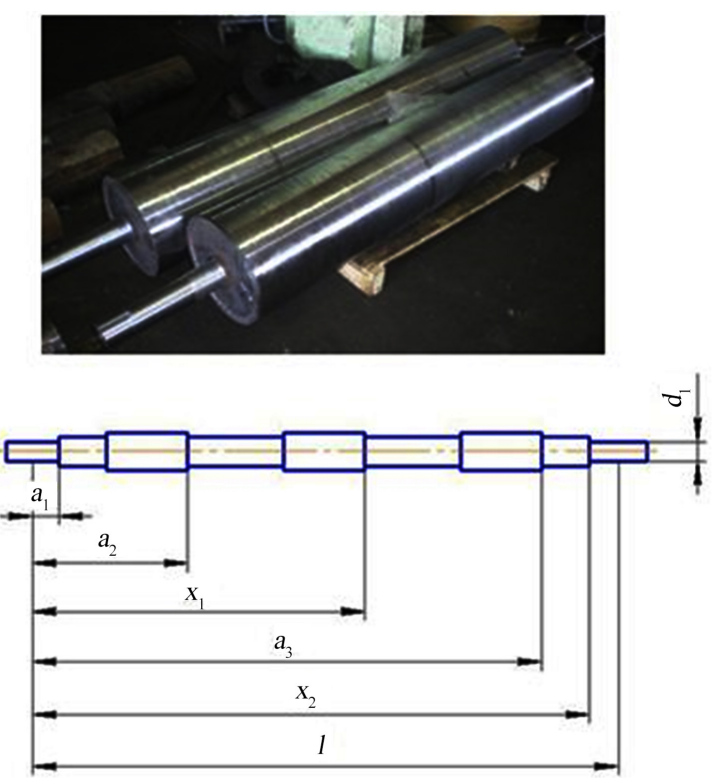

(b)

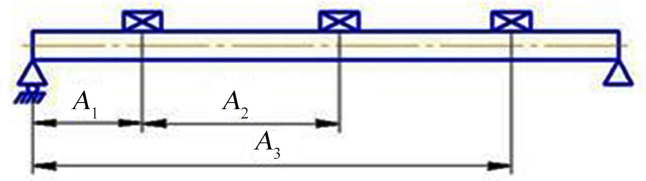

Figure 5. Scheme for calculating the critical rotation speed of the shaft. 
$q_{\text {equ }}$-the specific equivalent mass of the shaft;

$J_{\text {экв }}$-equivalent moment of inertia of the shaft and $m_{i}$ and $J_{i}$-details attached to the shaft and their moment of inertia about the free axis of the shaft;

l-the distance between the shaft supports.

In that case, the specific rotation frequency of the system:

$$
\rho=\sqrt{\frac{2 U_{O}}{L}}
$$

in this: $U_{O}$-the potential energy at the shear amplitude of the shaft

$$
U_{O}=\frac{1}{2} \int_{0}^{1} E J_{e q u}\left(\frac{\mathrm{d}^{2} y}{\mathrm{~d} X^{2}}\right)^{2} \mathrm{~d} x=f^{2} \frac{E J_{\text {equ }} \pi^{4}}{2 l^{4}} \int_{0}^{1} \sin ^{2} \frac{\pi x}{l} \mathrm{~d} x=f^{2} \frac{\pi^{4} E J_{\text {equ }}}{4 P}
$$

displacement of the first mass:

$$
y_{i}=f \sin \frac{\pi x_{i}}{l}
$$

rotation of the first mass:

$$
\theta=\left(\frac{\mathrm{d} y}{\mathrm{~d} x}\right)_{x=x_{i}}=-\frac{\pi}{l} f \cos \frac{\pi x_{i}}{l}
$$

Given the specific gravity of the shaft, the total shear amplitude and its deflection:

$$
\begin{aligned}
L & =\sum m_{i} y_{i}^{2}+\int_{0}^{1} q_{\text {equ }} y^{2} \mathrm{~d} x+\sum J_{i} \theta_{i}^{2} \\
& =f^{2}\left[\sum m_{i} \sin ^{2} \frac{\pi x_{i}}{l}+\frac{q_{\text {equ }} l}{2}+\frac{\pi^{2}}{l^{2}} \sum J_{i} \cos ^{2} \frac{\pi x_{i}}{l}\right]
\end{aligned}
$$

Substituting $U_{O}$ from Equation (13) and L from (16) into Equation (12) we obtain the following:

$$
\rho=\sqrt{\frac{2 \frac{\pi^{4} E J_{\text {equ }}}{4 d^{2}}}{\frac{q_{\text {equ }} l}{2}+\sum m_{i} \sin ^{2} \frac{\pi x_{1}}{l}+\frac{\pi^{2}}{l^{2}} \sum J_{i} \cos ^{2} \frac{\pi x_{i}}{l}}}
$$

To find a convenient solution to the equation, we introduce the following notation:

$$
\mu_{i}=\frac{m_{i}}{q_{\text {equ }} l}, x_{i}=\frac{J_{i}}{q_{\text {equ }} d^{3}}
$$

In that case we get the following

$$
\rho=\pi^{2} \sqrt{\frac{E J_{\text {equ }}}{q_{\text {equ }} l^{4}}} \cdot \sqrt{\frac{1}{1+2 \sum \mu_{i} \sin ^{2} \frac{J L x_{i}}{l}+2 \pi^{2} \sum x_{i} \cos ^{2} \frac{\pi x_{i}}{l}}}
$$

to solve the Equation (18) $J_{\text {зев }}$ and $q_{\text {экв }}$ will need to be found:

$$
J_{\text {зes }}=\left(J_{1}-J_{2}\right) \phi\left(\frac{a_{1}}{l}\right)+\left(J_{2}-J_{3}\right) \phi\left(\frac{a_{2}}{l}\right)+\cdots+\left(J_{n-1}-J_{n}\right) \phi\left(\frac{a_{n}-1}{l}\right)+J_{n}
$$




$$
q_{\text {экв }}=\left(q_{1}-q_{2}\right) \phi\left(\frac{a_{i}}{l}\right)+\left(q_{2}-q_{3}\right) \phi\left(\frac{a_{2}}{l}\right)+\cdots+\left(q_{n-1}-q_{n}\right) \phi\left(\frac{a_{n-1}}{l}\right)+q_{n}
$$

In Figure 6 a graph of the function $\phi\left(\frac{a}{l}\right)$.

Substituting the values $J_{e q u}$ and $q_{e q u}$ obtained into Equation (18), we can find the specific oscillation frequency of the shaft.

Considering the tensile stress along the shaft axis, it looks like this:

$$
\rho=\pi^{2} \sqrt{\frac{E J_{e q u}}{q l^{4}}} \cdot \sqrt{\frac{1}{1+2 \sum \mu_{i} \sin ^{2} \frac{\pi x_{i}}{l}+2 \pi^{2} \sum x_{i} \cos ^{2} \frac{\pi x_{i}}{l}}} \sqrt{1+\frac{A_{0}}{P_{k}}}
$$

in this: $A_{0}$-longitudinal force acting on the shaft;

$P_{k}$-the critical force acting on the shaft is the bending of the shaft corresponding to the plane of vibration of the shaft;

Determining the critical velocity in the transverse vibration of the saw drum shaft.

Saw drum shaft: Saw metal in the form of metal tape, cladding, washers and nuts under the influence of a generally equally distributed tension, the cantilever part of the shaft under the influence of gravity $G=100 H$ by the couplings and pulleys.

To perform the calculation, we use the following scheme [13] (Figure 7).

\section{Results}

The following is the data for the calculation:

Viscosity modulus of shaft material: $E=2.1 \times 10^{7}, \mathrm{~N} / \mathrm{cm}^{2}$

Ratio of clutch and pulley weight to equivalent shaft weight:

$$
\mu_{1}=\frac{G_{1}}{g q_{\text {equ }} l}=\frac{100}{980 \times 1.854 \times 10^{-2} \times 174}=0.574
$$

For calculations we accept the following:

For section 1:

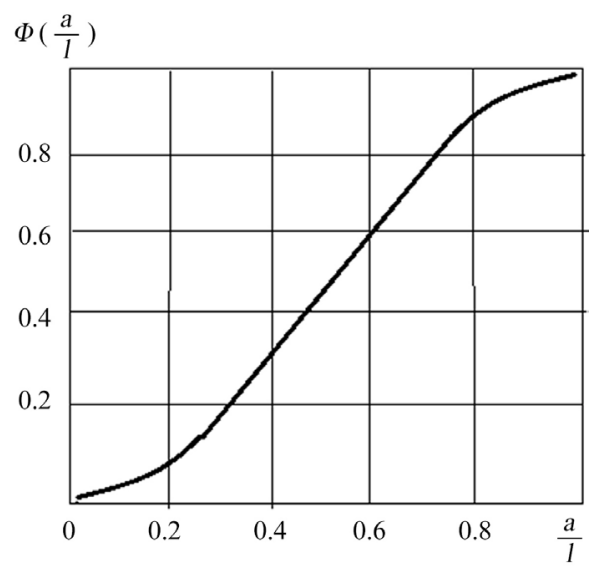

Figure 6. Graph of the function $\phi\left(\frac{a}{l}\right)$. 


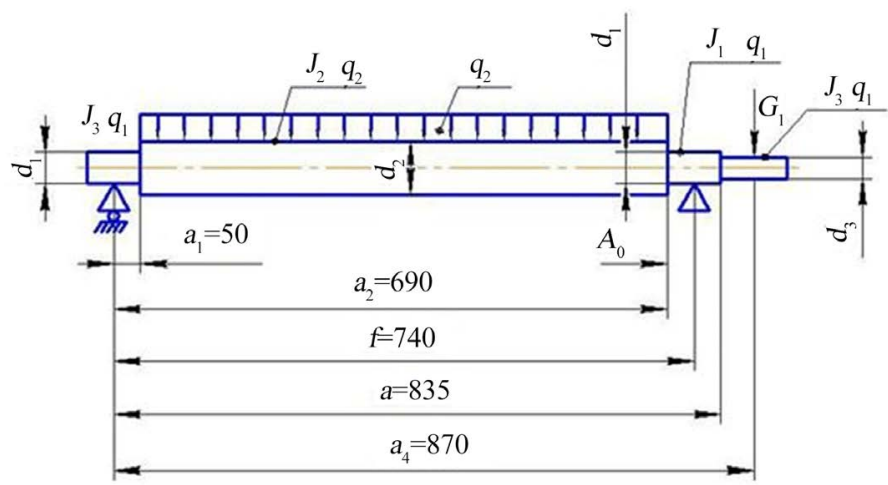

Figure 7. Scheme for determining the critical rotational speed of the drum shaft.

$$
\begin{aligned}
& d=6.0 \mathrm{~cm} ; F=23.7 \mathrm{~cm}^{2} ; q \times 10^{4}=0.189 \mathrm{~N} \cdot \frac{\mathrm{sec}^{2}}{\mathrm{~cm}^{2}} ; q_{i}-q_{i+1}=-1.366 ; \\
& J=44 \mathrm{~cm}^{4} ; J_{1}-J_{i+1}=44 ; \frac{a_{i}}{l}=0,00029 ; \phi\left(\frac{a_{i}}{l}\right)=0.0002 ; \\
& \left(q_{i}-q_{i+1}\right) \phi\left(\frac{a_{i}}{l}\right)=0.0002732 ;\left(J_{i}-J_{i+1}\right) \phi\left(\frac{a_{i}}{l}\right)=0.0894 ; \\
& \sum 1.554 \times 10^{2}=q_{\text {equ }} ; \sum 491=J_{\text {equ }} .
\end{aligned}
$$

For section 2:

$$
\begin{aligned}
& d=8.0 \mathrm{~cm} ; F=78.5 \mathrm{~cm}^{2} ; q \times 10^{4}=1.155 \mathrm{H} \cdot \frac{\mathrm{sec}^{2}}{\mathrm{~cm}^{2}} ; q_{i}-q_{i+1}=-1.366 \\
& J=44 \mathrm{~cm}^{4} ; J_{1}-J_{i+1}=44 ; \frac{a_{i}}{l}=0.972 ; \phi\left(\frac{a_{i}}{l}\right)=0.999 \\
& \left(q_{i}-q_{i+1}\right) \phi\left(\frac{a_{i}}{l}\right)=1.365 ;\left(J_{i}-J_{i+1}\right) \phi\left(\frac{a_{i}}{l}\right)=44.5
\end{aligned}
$$

For section 3:

$$
\begin{aligned}
& d=5.0 \mathrm{~cm} ; F=23.7 \mathrm{~cm}^{2} ; q \times 10^{4}=0.189 \mathrm{H} \cdot \frac{\mathrm{sec}^{2}}{\mathrm{~cm}^{2}} ; q_{i}-q_{i+1}=0.079 \\
& J=29.3 \mathrm{~cm}^{4} ; J_{1}-J_{i+1}=29.3 ; \frac{a_{i}}{l}=1.042 ; \phi\left(\frac{a_{i}}{l}\right)=1.007 \\
& \left(q_{i}-q_{i+1}\right) \phi\left(\frac{a_{i}}{l}\right)=0.079 ;\left(J_{i}-J_{i+1}\right) \phi\left(\frac{a_{i}}{l}\right)=29.4
\end{aligned}
$$

For section 4:

$$
\begin{aligned}
& d=4.0 \mathrm{~cm} ; F=13.7 \mathrm{~cm}^{2} ; q \times 10^{4}=0.110 \mathrm{H} \cdot \frac{\mathrm{sec}^{2}}{\mathrm{~cm}^{2}} ; q_{i}-q_{i+1}=0.110 \\
& J=15.3 \mathrm{~cm}^{4} ; J_{1}-J_{i+1}=15.3 ; \frac{a_{i}}{l}=1.104 ; \phi\left(\frac{a_{i}}{l}\right)=1.007 \\
& \left(q_{i}-q_{i+1}\right) \phi\left(\frac{a_{i}}{l}\right)=0.110 ;\left(J_{i}-J_{i+1}\right) \phi\left(\frac{a_{i}}{l}\right)=153 \\
& \rho=\pi^{2} \sqrt{\frac{2.1 \times 10^{7} \times 49.1}{1.554 \times 10^{-2} \times 1.74 \times 10^{8}}} \sqrt{\frac{1+0.248 \sin ^{2} \frac{3.14 \times 157}{174}}{1+174}}=63 \frac{1}{\mathrm{sec}}
\end{aligned}
$$


In that case: $V_{k r}=\frac{P}{2 \pi}=\frac{63}{6.28}=10.3 \mathrm{Gt}$

From this: $n_{k r}=v_{k r} \times 60=10.3 \times 60=618 \mathrm{rpm}$

Given the tensile force along the shaft axis of the saw drum: $A_{0}=2000 \mathrm{~N}$

$$
n_{k r}^{\prime}=n_{k r} \sqrt{1+\frac{A_{0}}{P_{K}}}=618 \sqrt{1+\frac{2000}{348620}}=618 \times 1.003=620 \mathrm{rpm}
$$

As a result of calculating the resistance of the shaft to stiffness and vibration (Figure 8), it was determined that the shafts are resistant to vibration as a result of periodic loading.

Based on this study, a graph of the relationship of the critical rotational speed of the shaft to the oscillation frequency along its axis was obtained (Figure 9). The frequency of oscillation along its axis was determined by the critical rotational frequency of the shaft, and it is $v_{k r}=10.3 \mathrm{Gt}$.

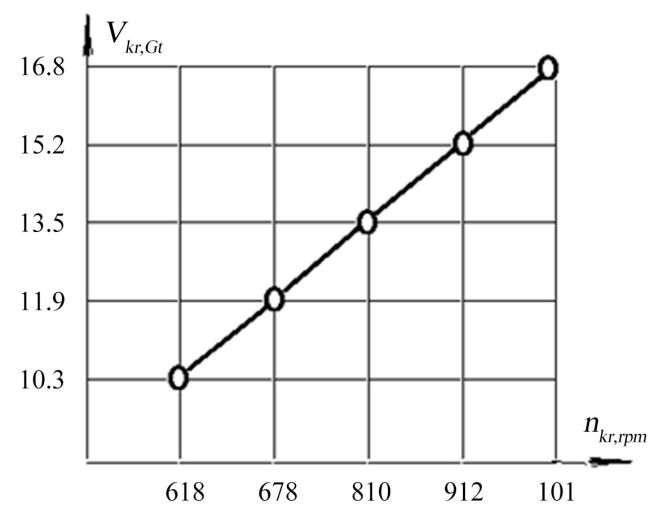

Figure 8. Graph of the relationship of the critical rotational speed of a shaft to the oscillation frequency along its axis.

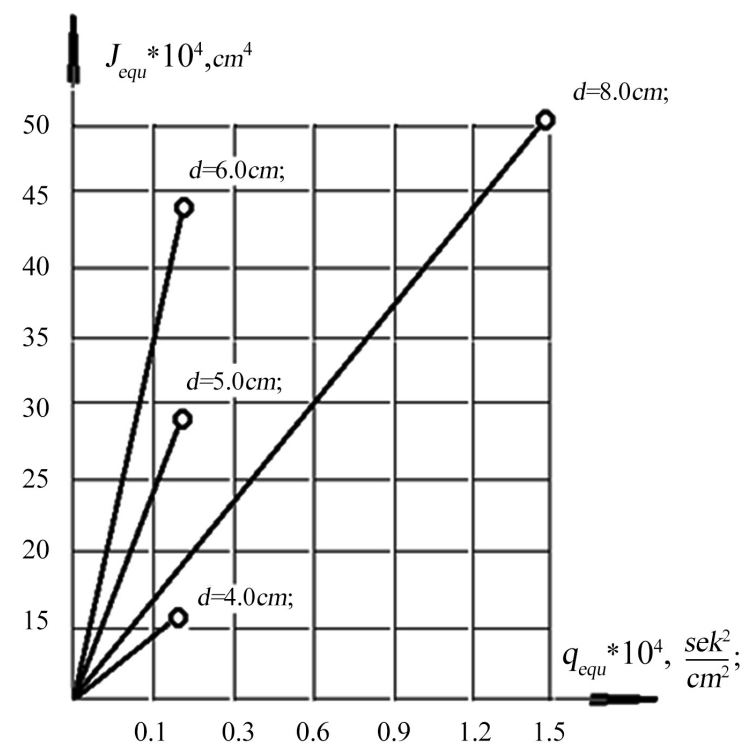

Figure 9. Graph of the relationship of the specific mass of an equivalent shaft at different diameters of the shaft to the equivalent moment of inertia. 


\section{Conclusions}

As a result of calculating the strength of the saw drum, it is a steel coating that is under more stress, and it was found that the strength reserve of the drum is $\left[\delta_{T}\right]$ $=2.03$ ( where $\delta_{T}=0.8-2.5$ ).

By calculating the resistance of the saw drum shaft to stiffness and vibration, it was determined that the shafts are resistant to vibration as a result of periodic loading and that the frequency of vibration along its axis through the critical rotation frequency is $v_{k r}=10.3 \mathrm{Gt}$.

\section{Conflicts of Interest}

The authors declare no conflicts of interest regarding the publication of this paper.

\section{References}

[1] Budanov, K.D., Martirosov, A.A., Popov, E.A., et al. (1975) Fundamentals of Theory, Design and Calculation of Textile Machines. Mashinostroenie, $390 \mathrm{p}$.

[2] Sultanov, M. and Obidov, A. (2020) Investigation of Working Parts of Fixed Device Designed to Separate Spinning Fibers from Fibrous Waste That Can Be Spun. Journal of Critical Reviews (JCR), 7, 2314-2322.

[3] Avazbek, O., Mirzaolim, S., Ibrohim, M. and Shokir, A. (2018) The Theoretical Studies of the Cultivation of Three Cotton Seeds along the Plain. Engineering, 10, 514-520. https://doi.org/10.4236/eng.2018.108037

[4] Obidov, A.A. and Sultanov, M.M. (2020) To Research the Method of Separating Fibers Suitable for Spinning on a Needle Drum. International Scientific and Practical Conference Cutting Edge-Science, 29-30 June 2020, Shawnee, 128-131.

[5] Sultonov, M.M. and Obidov, A.A. (2020) Study of Fiber Motion in a Needle Drum Fiber Separation Device. Universum: Technical Science, 33-37.

[6] Obidov, A.A. and Sultanov, M.M. (2020) Study of Technological Parameters of Fiber Separation Device. International Journal of Psychosocial Rehabilitation, 24, 6400-6407. https://doi.org/10.37200/IJPR/V24I5/PR2020624

[7] Utkirbek, B., Azamatovich, O.A., Juraboyevich, M.K. and Mirzarahmatovich, S.M. (2019) Research Capacity of the Fiber of Long Fiber Separating Drums from Waste Fibers Composition. International Journal of Innovative Studies in Sciences and Engineering Technology (IJISSET), 5, 28-31.

[8] Ashnin, P.N. (1985) Carding of Fibrous Materials. Light Industry and Consumer Services, 320-325.

[9] Obidov, A.A., Goyibnazarov, E.E. and Abdullaev, R. (2015) Extraction of Long Fibers from Fibrous Wastes. NamMTI, May 2015.

[10] Sevostyanov, A.G. (2007) Methods and Means of Research of Mechanical and Technological Processes of the Textile Industry. Moscow State Technical University Named after Kosygin. LLC "Soviage Bevo".

[11] Obidov, A., Mamatqulov, O. and Sultanov, M. (2018) Theoretical Analysis of the Movement of Cotton Piece on the Slope Surface. International Conference of Science and Practice: A New Level of Integration in the Modern World, Berlin, 151-156.

[12] Miroshnichenko, G.I. (1972) Fundamentals of Designing Machines for Primary Pro- 
cessing of Cotton. Mechanical Engineering, 337.

[13] Obidov, A.A. (2006) Investigation of the Working Surface of a New Seeding Machine. Journal of Textile Problems, 2, 29-32. 